\title{
Wave effect on the trajectory of a high-speed rigid body in a water column
}

\author{
P. C. Chu \& C. Fan \\ Naval Ocean Analysis and Prediction Laboratory Naval \\ Postgraduate School, Monterey, California, USA
}

\begin{abstract}
Waves form when the water surface is disturbed, for example, by wind or gravitational forces. During such disturbances energy and momentum are transferred to the water mass and sea-state is changed. For shallow water regions, the bottom topography affects the waves dramatically. Waves may cause an evident slant of the ocean surface with a large inclination angle of about $55^{\circ}$. When a high-speed rigid body strikes the wavy ocean surface, the random ocean slope largely affects its underwater trajectory. Identification of such an effect is a key point in the prediction of a fast moving rigid body in a water column. The probability density equation (PDF) of the ocean wave slopes was used to generate the stochastic surface slope. A 6-degree of freedom (DOF) model of a fast-moving rigid body is used with the stochastic sloping surface as its waterentry condition. The PDFs of the body location and orientation are obtained from results of 6-DOF model runs. From temporal change of these PDFs, the wave effect on the trajectory of a high-speed underwater rigid body has been identified.
\end{abstract}

Keywords: body-flow interaction, 3D bomb trajectory prediction, 6-DOF model, STRIKE35, random waves, probability density function.

\section{Introduction}

Movement of a fast-moving rigid body such as a bomb through a water column has been studied recently [1-3]. These studies have been motivated by a new concept of using a general purpose bomb such as the Mk-84 for mine/maritime improvised explosive device (IED) clearance in order to reduce the risk to 
BOMB FALL LINE

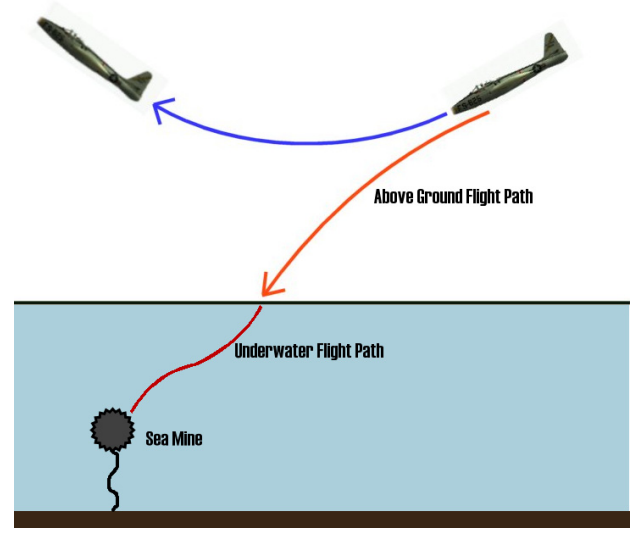

Figure 1: The concept of airborne sea mine/maritime IED clearance.

personnel and to decrease the sweep timeline without sacrificing effectiveness (Fig. 1). The horizontal distance $(r)$ (or called trajectory deviation) between surface impact point and the bomb location varies with depth in different types of trajectories. This parameter draws attention to the naval research due to the threat of mine and maritime IED. Prediction of trajectory deviation of an underwater bomb contributes to the bomb breaching for mine and maritime IED clearance in surf and very shallow water zones with depth shallower than $12.2 \mathrm{~m}$ (i.e., $40 \mathrm{ft}$ ), shallow water zones $(12.2-91.4 \mathrm{~m}$, i.e., $40-300 \mathrm{ft})$, and deep zones (deeper than $91.4 \mathrm{~m}$, i.e., $300 \mathrm{ft}$ ) according to U.S. Navy's standards. The bombs' trajectory drift is required to satisfy the condition, $r \leq 2.1 \mathrm{~m}$, for the validity of mine clearance using bombs [5].

In coastal oceans, waves form when the water surface is disturbed, for example, by wind or gravitational forces. During such disturbances energy and momentum are transferred to the water mass and sea-state is changed. For very shallow and shallow water regions, the bottom topography affects the waves dramatically and causes a significant change in surface slope. When bomb strikes on the wavy ocean surface, a scientific problem arises: How does randomly changing ocean-surface slope affect the underwater bomb trajectory and orientation? Or what is the probability density function of the underwater bomb trajectory deviation due to random sea surface slope? The major task of this paper is to answer these questions. Stochastic features of the sea slope are simply described in Section 2. A recently developed six degrees of freedom (6DOF) model at the Naval Postgraduate School for predicting underwater bomb location and trajectory is depicted in Section 3. Ensemble 6-DOF modelling to get PDF of trajectory deviation from the stochastically changing sea surface slope is described in Section 4. The conclusions are presented in Section 5. 


\section{PDF of ocean surface slope}

Wave height and wave period are approximately independent of each other for either wind waves or swells, but not for mixed waves. From mixed wave records, Gooda [8] found that there is a strong correlation between wave height and wave period. In fact, the correlation is mainly caused by the two or more groups of notable waves with different characteristic wave heights and periods in the mixed waves. With the independent assumption between wave amplitude and wave period (or wavelength), the PDF of averaged wave slope $s$ scaled by its standard deviation $\sigma$ (the real slope is $\mathrm{s}^{*}=\mathrm{s} \sigma$ ) is obtained from the PDF of wave length and PDF of wave amplitude [9],

$$
p(s)=\frac{n}{(n-1)} s\left[1+\frac{s^{2}}{(n-1)}\right]^{-(n+2) / 2},
$$

where $n$ is the peakedness coefficient which is determined by both the spectral width of the gravity waves, and the ratio between the gravity wave mean-square slope and the detectable short wave mean-square slope. Generally speaking, the peakedness of slopes is generated by nonlinear wave-wave interactions in the range of gravity waves; and the skewness of slopes is generated by nonlinear coupling between the short waves and the underlying long waves. For $n=2$, the PDF of the wavelength corresponds to the Rayleigh distribution. For $n=10$, the PDF in (4) fits the Gram Charlier distribution [10], very well in the range of small slopes. As $n \rightarrow \infty$, the PDF of the wavelength tends to the Gaussian distribution [9]. Fig. 2 shows four typical surface-slope characteristics: (a) $n=2$, (b) $n=4$, (c) $n=10$, and $n=100$. It is seen that There is almost no difference in PDF between $n=10$ and $n=100$.
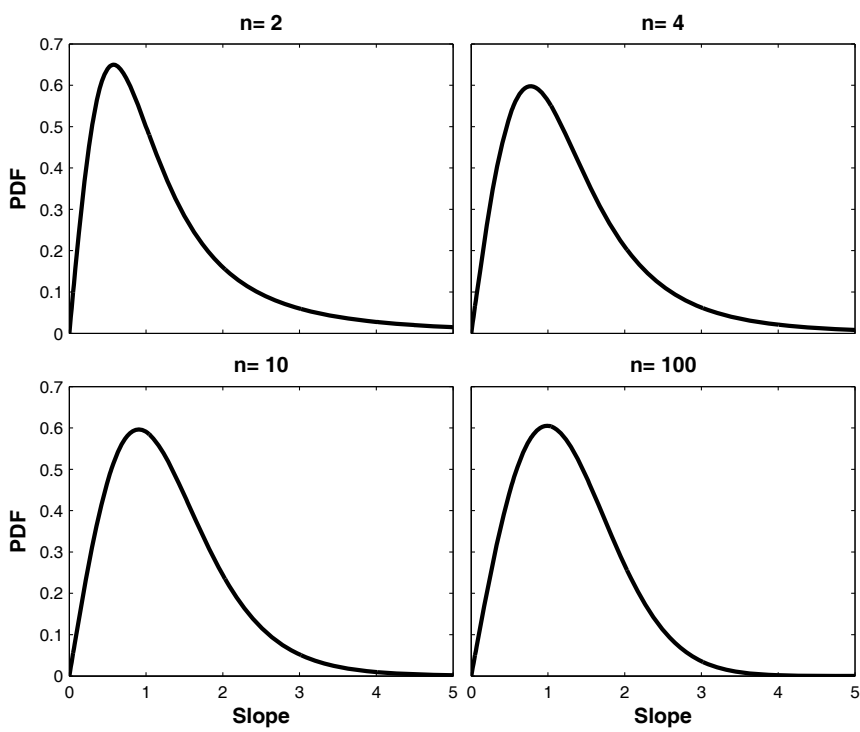

Figure 2: The s-PDFs for various surface characteristics: (a) $n=2$, (b) $n=4$, (c) $n=10$, and (d) $n=100$. 


\section{A 6-DOF model (STRIKE35)}

Recently, a 6-DOF model has been developed at the Naval Postgraduate School for predicting underwater bomb location and trajectory. It contains three parts: momentum equation, moment of momentum equation, and semi-empirical formulas for drag, lift, and torque coefficients [11-13]. The momentum equation of a rigid body is given by

$$
m \frac{d \mathbf{u}}{d t}=\mathbf{F}_{g}+\mathbf{F}_{b}+\mathbf{F}_{d}+\mathbf{F}_{l},
$$

where $m$ is the mass of the rigid body, $\mathbf{u}$ is the translation velocity of the centre of mass,

$$
\mathbf{F}_{g}=-m g \mathbf{k}, \quad \mathbf{F}_{b}=\rho \Pi g \mathbf{k},
$$

are the gravity and buoyancy force; $\Pi$ is the volume of the rigid body; $\mathbf{k}$ is the unit vector in the vertical direction (positive upward): and $\mathrm{g}$ is the gravitational acceleration. $\mathbf{F}_{d}$ is the drag force; and $\mathbf{F}_{l}$ is the lift force. The moment of momentum equation is given by

$$
\mathbf{J} \bullet \frac{d \mathbf{\Omega}}{d t}=-\sigma \mathbf{e} \times(\rho \Pi g \mathbf{k})+\mathbf{M}_{h},
$$

where $\boldsymbol{\Omega}$ is the rigid-body's angular velocity vector; $\sigma$ is the distance between centre of volume $\left(o_{v}\right)$ and centre of mass $\left(o_{m}\right)$, which has a positive (negative) value when the direction from $o_{v}$ to $o_{m}$ is the same (opposite) as the unit vector $\mathbf{e} ; \mathbf{M}_{\mathrm{h}}$ is the hydrodynamic torque due to the drag/lift forces; and $\mathbf{J}$ is the gyration tensor.

The drag/lift/torque coefficients should be given before running the 6-DOF model. These coefficients depend on various physical processes such as water surface penetration, super-cavitation, and bubble dynamics. A diagnosticphotographic method has been developed [4] to get semi-empirical formulae for calculating the drag/lift/torque coefficients for underwater bombs with dependence on the Reynolds number $(\mathrm{Re})$, angle of attack $(\alpha)$, and rotation rate along the bomb's major axis $(\Omega)[4]$,

$$
\begin{gathered}
C_{d}=0.02+0.35 e^{-2(\alpha-\pi / 2)^{2}}\left(\frac{\mathrm{Re}}{\mathrm{Re}^{*}}\right)^{0.2}+0.008 \Omega \sin \theta, \\
C_{l}= \begin{cases}0.35 \sin \left(\theta_{1}\right)\left(\frac{\mathrm{Re}}{\mathrm{Re}^{*}}\right)^{0.2} & \text { if } \alpha \leq \frac{\pi}{2} \\
0.1 \sin \left(\theta_{2}\right)-0.015 \Omega\left(\frac{\mathrm{Re}}{\mathrm{Re}^{*}}\right)^{2} \sin \left(\theta_{2}^{0.85}\right) & \text { if } \alpha>\frac{\pi}{2}\end{cases}
\end{gathered}
$$




$$
C_{m}=\left\{\begin{array}{cc}
0.07 \sin (2 \alpha)\left(\frac{\mathrm{Re}^{*}}{\mathrm{Re}}\right)^{0.2} & \text { if } \alpha \leq \frac{\pi}{2} \\
0.02 \sin (2 \alpha) \sqrt{\left(\frac{\mathrm{Re}}{\mathrm{Re}^{*}}\right)} & \text { if } \alpha>\frac{\pi}{2}
\end{array}\right.
$$

Here, $\operatorname{Re}^{*}=1.8 \times 10^{7}$, is the critical Reynolds number, and

$$
\begin{gathered}
\theta \equiv\left(\pi^{2.2}-(\pi-|\pi-2 \alpha|)^{2.2}\right)^{\frac{1}{2.2}} \operatorname{sign}(\pi-2 \alpha), \\
\theta_{1}=\pi\left(\frac{2 \alpha}{\pi}\right)^{1.8}, \theta_{2}=2 \pi\left(\frac{2 \alpha}{\pi}-1\right)^{0.7} .
\end{gathered}
$$

\section{PDF of bomb's horizontal drift}

Let the bomb be dropped in the vertical direction to the slanted sea surface characterized by an averaged slope $\left(s^{*}=\sigma s\right)$ in a wave period, here $s^{*}=\tan \mu$ (see Fig. 3). Consider a 5-time of $\mathrm{s}^{*}$ value as the interval [0,5s*] for the change of the surface slope. This interval $\left[0,5 \mathrm{~s}^{*}\right]$ is divided into $I$ equal sub-intervals,

$$
\sigma s_{i}=\frac{5 i s^{*}}{I}, i=0,1,2, \ldots, I,
$$

with the corresponding inclination,

$$
\mu_{i}=\arctan \left(\sigma s_{i}\right)=\arctan \frac{5 i s^{*}}{I}, i=0,1,2, \ldots, I .
$$

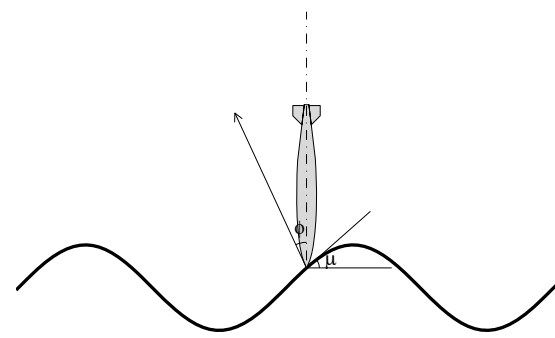

Figure 3: Ocean surface inclination angle $(\mu)$ and bomb impact angle $(\varphi)$ relative to the normal direction of the surface.

For a given parameter $n$ in the $s-\mathrm{PDF}$, the probability for $s^{*}$ taking values between $\sigma s_{i-1}$ and $\sigma s_{i}$ is calculated by

$$
P_{i} \equiv \operatorname{Prob}\left(s_{i} \leq s \leq s_{i+1}\right)=\int_{s_{i}}^{s_{i+1}} p(s) d s .
$$


The 6-DOF model is integrated $I$ times (called ensemble integration) from the surface impact speed $(V)$ and various $\mu_{\mathrm{i}}$ values to get the bomb horizontal drift $\hat{r}_{i}$ $(i=0,1, \ldots, I)$ at depth $z=-H$. The series $\left\{\hat{r}_{i}, i=0,1, \ldots, I\right\}$ might not be in monotonically increasing or decreasing order. Therefore, it is reorganized into monotonically increasing order $\left\{r_{j}, j=0,1, \ldots, J\right\}$ with $J \leq I$. The inequality is due to an interval $\left[r_{j}, r_{j+1}\right]$ of the horizontal drift corresponding to $m$ intervals $\left\{\left[s_{i 1}, s_{i 1}+1\right],\left[s_{i 2}, s_{i 2}+1\right], \ldots,\left[s_{i m}, s_{i m}+1\right]\right\}$ of the surface slope (Fig. 4).

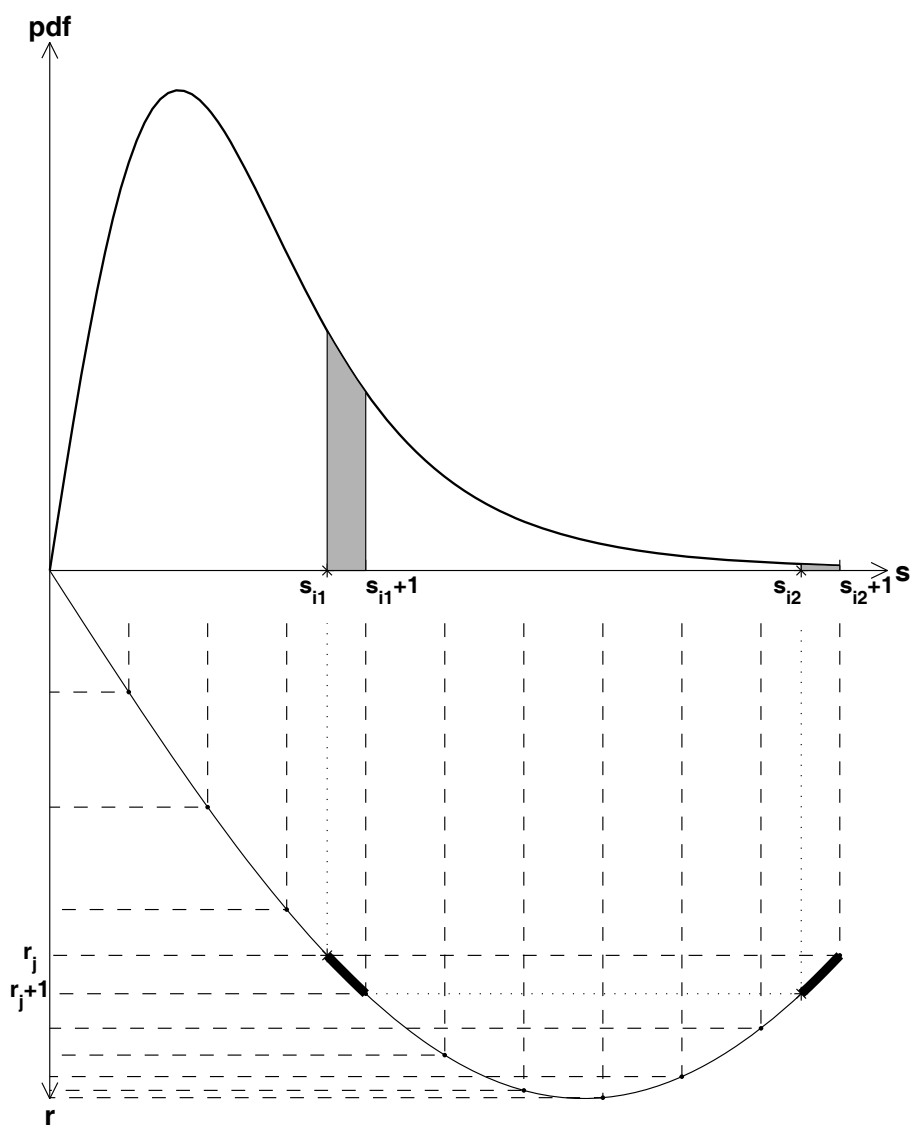

Figure 4: Calculation of the probability for the bomb's horizontal drift $r$ taking values between $r_{j}$ and $r_{j+1}$ from $m$ intervals of surface slope s. Here, $m=1$, and $m=2$.

The probability for the bomb's horizontal drift $r$ taking values between $r_{j}$ and $r_{j+1}$ is calculated by

$$
Q_{j} \equiv \operatorname{Prob}\left(r_{j} \leq r \leq r_{j+1}\right)=\int_{s_{i 1}}^{s_{i 1+1}} p(s) d s+\int_{s_{i 2}}^{s_{i 2+1}} p(s) d s+\ldots+\int_{s_{i n}}^{s_{i n+1}} p(s) d s
$$


The probability density between $r_{j}$ and $r_{j+1}$ is calculated by

$$
p_{j}=\frac{Q_{j}}{r_{j+1}-r_{j}} .
$$

From $p_{j}$, we can obtain the PDF of $r$, or called the $r$-PDF. Dependence of $r$ PDF on depth can be identified from the ensemble integration $(I=100)$ of the 6DOF model with given bomb's surface impact speed $(V=300 \mathrm{~m} / \mathrm{s}), s^{*}=0.2$ (i.e., $\sigma=0.2$ ), and $n=2$ (i.e., large peakedness in the $s$-PDF). The calculated $r$ PDF (Fig. 5) is positively skewed for shallow depth $(H=12.2 \mathrm{~m}$, i.e., $40 \mathrm{ft})$, reduces the skewness as depth increases to $50 \mathrm{~m}$, becomes negatively skewed as the depth exceeding $91.4 \mathrm{~m}$ (i.e., $300 \mathrm{ft}$ ).
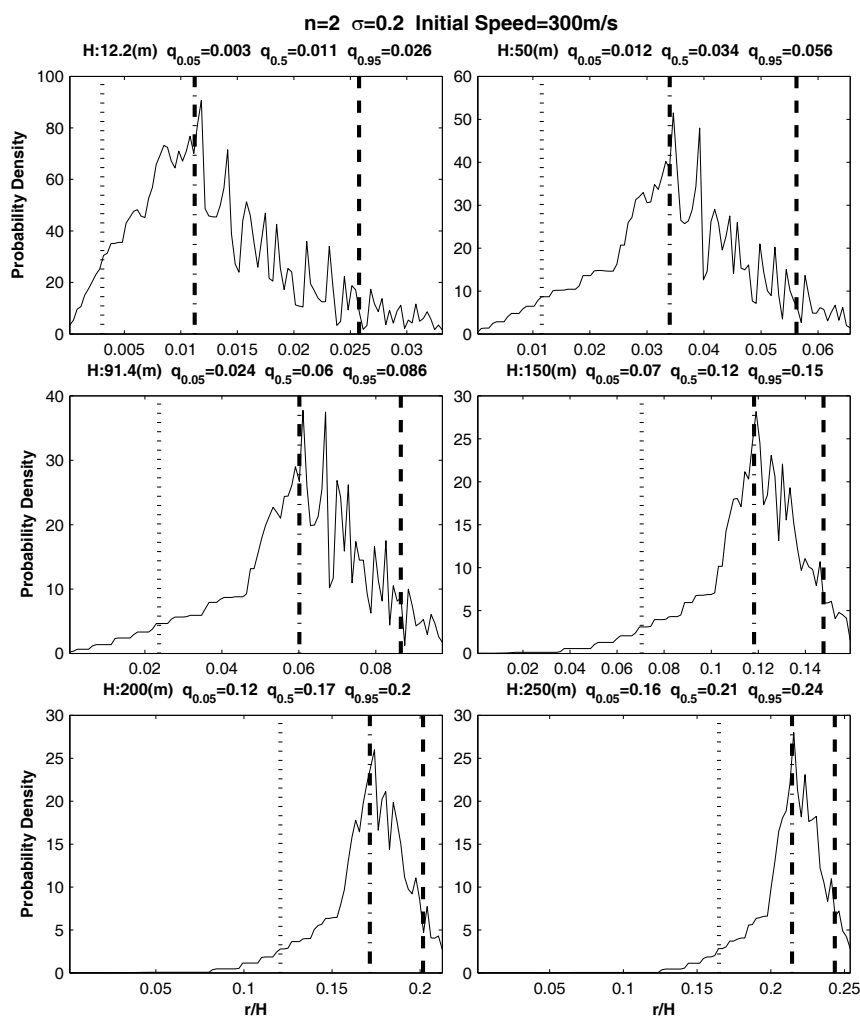

Figure 5: Probability distribution of the bomb's horizontal drift (scaled by the depth) $r / H$ with $n=2, \sigma=0.2$, and $V=300 \mathrm{~m} / \mathrm{s}$ for various depth: (a) $12.2 \mathrm{~m}$ (i.e. $40 \mathrm{ft}$ ), (b) $50 \mathrm{~m}$, (c) $91.4 \mathrm{~m}$ (i.e., $300 \mathrm{ft}$ ), (d) $150 \mathrm{~m}$, (e) $200 \mathrm{~m}$, and (f) $250 \mathrm{~m}$. 
The negative skewness strengthens as depth deeper than $91.4 \mathrm{~m}$. The horizontal axis in all the panels Fig. 5 is the non-dimensional horizontal drift $r / H$. The median (50 percentile $q_{0.5}$ ) of the horizontal drift $(r)$ is $0.16 \mathrm{~m}$ at the depth

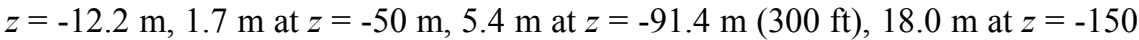
$\mathrm{m}, 34.0 \mathrm{~m}$ at $z=-200 \mathrm{~m}$, and $52.5 \mathrm{~m}$ at $z=-250 \mathrm{~m}$ (Table 1). Here $z$ is the vertical coordinates with $z=0$ corresponding to the water surface. Thus, down to the depth of $50 \mathrm{~m}$, the median value of the horizontal drift is always less than the Navy's criterion, i.e., $2.1 \mathrm{~m}$. The 95 percentile $\left(q_{0.95}\right)$ of the horizontal drift $(r)$ represents a reasonable estimation (with $95 \%$ of confidence) of the distance between bomb and mine/maritime IED when the bomb maneuvering in the water column. If this value is smaller than $2.1 \mathrm{~m}$, according to the Navy's standard, the bomb will effectively 'kill' the mine/maritime IED. It is $0.32 \mathrm{~m}$ at the depth $z=-12.2 \mathrm{~m}, 2.8 \mathrm{~m}$ at $z=-50 \mathrm{~m}, 7.86 \mathrm{~m}$ at $z=-91.4 \mathrm{~m}(300 \mathrm{ft}), 22.5 \mathrm{~m}$ at $z=-150 \mathrm{~m}, 40.0 \mathrm{~m}$ at $z=-200 \mathrm{~m}$, and $60.0 \mathrm{~m}$ at $z=-250 \mathrm{~m}$ (Table 2). The 5 percentile $\left(q_{0.05}\right)$ of the horizontal drift $(r)$ represents the minimum distance (likely) between bomb and mine/maritime IED when the bomb maneuvering in

Table 1: The median horizontal drift (unit: $m$ ) of an underwater bomb at various depths obtained from ensemble integration of the 6-DOF model with various input parameters.

\begin{tabular}{|c|c|c|c|c|}
\hline Depth $(\mathrm{m})$ & Case 1: & Case 2: & Case 3: & Case 4: \\
& $V=300 \mathrm{~m} / \mathrm{s}$ & $V=300 \mathrm{~m} / \mathrm{s}$ & $V=300 \mathrm{~m} / \mathrm{s}$ & $V=200 \mathrm{~m} / \mathrm{s}$ \\
& $n=2$ & $n=100$ & $n=2$ & $n=2$ \\
& $\sigma=0.2$ & $\sigma=0.2$ & $\sigma=1.0$ & $\sigma=0.2$ \\
\hline 12.2 & 0.16 & 0.16 & 0.37 & 0.17 \\
\hline 50.0 & 1.7 & 1.8 & 3.1 & 2.5 \\
\hline 91.4 & 5.4 & 5.7 & 8.6 & 8.9 \\
\hline 150.0 & 18.0 & 18.0 & 22.5 & 25.5 \\
\hline 200.0 & 34.0 & 34.0 & 42.0 & 44.0 \\
\hline 250.0 & 52.5 & 55.0 & 62.5 & 65.0 \\
\hline
\end{tabular}

Table 2: The values of $q_{0.95}$ for the horizontal drift (unit: $m$ ) of an underwater bomb at various depths obtained from ensemble integration of the 6-DOF model with various input parameters.

\begin{tabular}{|c|c|c|c|c|}
\hline Depth (m) & Case 1: & Case 2: & Case 3: & Case 4: \\
& $V=300 \mathrm{~m} / \mathrm{s}$ & $V=300 \mathrm{~m} / \mathrm{s}$ & $V=300 \mathrm{~m} / \mathrm{s}$ & $V=200 \mathrm{~m} / \mathrm{s}$ \\
& $n=2$ & $n=100$ & $n=2$ & $n=2$ \\
& $\sigma=0.2$ & $\sigma=0.2$ & $\sigma=1.0$ & $\sigma=0.2$ \\
\hline 12.2 & 0.32 & 0.27 & 0.54 & 0.17 \\
\hline 50.0 & 2.8 & 2.55 & 4.0 & 3.6 \\
\hline 91.4 & 7.86 & 7.40 & 10.05 & 10.97 \\
\hline 150.0 & 22.5 & 21.0 & 25.5 & 28.5 \\
\hline 200.0 & 40.0 & 38.0 & 46.0 & 48.0 \\
\hline 250.0 & 60.0 & 60.0 & 67.5 & 70.0 \\
\hline
\end{tabular}


Table 3: The values of $q_{0.05}$ for the horizontal drift (unit: $\mathrm{m}$ ) of an underwater bomb at various depths obtained from ensemble integration of the 6-DOF model with various input parameters.

\begin{tabular}{|c|c|c|c|c|}
\hline Depth (m) & Case 1: & Case 2: & Case 3: & Case 4: \\
& $V=300 \mathrm{~m} / \mathrm{s}$ & $V=300 \mathrm{~m} / \mathrm{s}$ & $V=300 \mathrm{~m} / \mathrm{s}$ & $V=200 \mathrm{~m} / \mathrm{s}$ \\
& $n=2$ & $n=100$ & $n=2$ & $n=2$ \\
& $\sigma=0.2$ & $\sigma=0.2$ & $\sigma=1.0$ & $\sigma=0.2$ \\
\hline 12.2 & 0.13 & 0.05 & 0.15 & 0.04 \\
\hline 50.0 & 0.6 & 0.80 & 1.8 & 1.05 \\
\hline 91.4 & 5.48 & 7.40 & 5.76 & 5.30 \\
\hline 150.0 & 10.5 & 12.45 & 18.0 & 18.0 \\
\hline 200.0 & 24.0 & 26.0 & 34.0 & 32.0 \\
\hline 250.0 & 40.0 & 45.0 & 55.0 & 55.0 \\
\hline
\end{tabular}

the water column. It is $0.13 \mathrm{~m}$ at the depth $z=-12.2 \mathrm{~m}, 0.6 \mathrm{~m}$ at $z=-50 \mathrm{~m}$, $5.48 \mathrm{~m}$ at $z=-91.4 \mathrm{~m}(300 \mathrm{ft}), 10.5 \mathrm{~m}$ at $z=-150 \mathrm{~m}, 24.0 \mathrm{~m}$ at $z=-200 \mathrm{~m}$, and $40.0 \mathrm{~m}$ at $z=-250 \mathrm{~m}$ (Table 3 ).

\section{Conclusions}

The PDF of the horizontal drift of underwater bomb trajectory (i.e., $r$-PDF) due to stochastic ocean surface slope is obtained through ensemble integration of the 6-DOF model recently developed at the Naval Postgraduate School. For a bomb dropping in the vertical direction to a slanted sea surface, the input parameters of the 6-DOF model are the bomb's surface impact speed $(V)$, and surface slope. The surface slope is a random variable depending on two parameters: (a) averaged slope within a wave period $(\sigma)$, and $(b)$ peakedness of the $s-\operatorname{PDF}(n)$.

The s-PDF is discretized into $I$ intervals (in this paper, $I=100$ ). For given values of $(V, \sigma, n)$, the 6-DOF model is integrated $I$ times with different values of the surface slope from the $s$-PDF to obtained $I$ values of the horizontal drift at various depth. The $r$-PDF is then constructed from these $r$ values. The r-PDF has the following features:

(1) The $r$-PDF varies with depth. Usually, the $r$-PDF is positively skewed for very shallow water $(H=12.2 \mathrm{~m}$, i.e., $40 \mathrm{ft})$, and negatively skewed down below. Increase of the peakedness parameter of the $s$-PDF $(n)$ or the averaged surface slope in a wave period $(\sigma)$ reduces the positive skewness at the very shallow water and enhances the negative skewness. Decrease of the bomb's surface impact speed $(V)$ enhances the peakedness of the $r$-PDF. Three measures were calculated $\left(q_{0.05}, q_{0.5}\right.$, and $\left.q_{0.95}\right)$ from the $r$-PDF.

(2) The values of $q_{0.95}$ are small for all cases at a very shallow depth $(\mathrm{z}=-12.2 \mathrm{~m}$, i.e., $40 \mathrm{ft})$ with a maximum value of $0.54 \mathrm{~m}$ for the initial conditions of $(V=300 \mathrm{~m} / \mathrm{s}, n=2, \sigma=1.0)$. This value $(0.54 \mathrm{~m})$ is much smaller than the critical value of $2.1 \mathrm{~m}$ for effectively ' $\mathrm{killing}$ ' the mine/maritime. 
(3) The values of $q_{0.95}$ are all larger than $2.1 \mathrm{~m}$ when the depth deeper than $50 \mathrm{~m}$. This indicates that to extend the bomb breaching technology from very shallow water $(12.2 \mathrm{~m}$ depth) to shallow water $(12.2 \mathrm{~m}-91.4 \mathrm{~m})$ needs more studies.

\section{Acknowledgements}

The Office of Naval Research Breaching Technology Program (Grant Number: N0001410WX20165, Program Manager: Brian Almquist) supported this study. The authors thank Mr. Kennard Watson from the Naval Surface Warfare Center Panama City Division for very useful comments and helps.

\section{References}

[1] Chu, P.C., and G. P. Ray, 2006, "Prediction of high-speed rigid body maneuvering in air-water-sediment," Adv. Fluid Mech., 6, edited by M. Rahman and C.A. Brebbia, WIT Press (ISBN-1-84564-163-9), 43-52.

[2] Ray G. P., 2006. Bomb Strike Experiments for Mine Clearance Operations. MS Thesis in Meteorology and Physical Oceanography, Naval Postgraduate School, Monterey, California, pp. 197.

[3] Chu, P.C., Fan, C.W., and Gefken, P.R., 2008, "Semi-empirical formulas of drag/lift coefficients for high-speed rigid body maneuvering in water column," Adv. Fluid Mech., 7, edited by M. Rahman and C.A. Brebbia, WIT Press (ISSN-1743-3533), 163-172.

[4] Chu, P.C., Fan, C.W., and P. R. Gefken, 2010. "Diagnostic-photographic determination of drag/lift/torque coefficients of high speed rigid body in water column," ASME J. Appl. Mech., 77, 011015-1- 011015-15.

[5] Humes, G., 2007. Technology Transition Agreement, EC SHD-FYO6-03 FNC Product: Standoff Assault Breaching Weapon Fuze Improvement. pp. 10.

[6] Kinsman, B., 1965, Wind Waves, Prentice-Hall Inc., Englewood Cliffs, New Jersey, Library of Congress Catalog Card Number: 64-10136, pp. 676.

[7] Dare, A., Landsberg, A., Kee, A., and Wardlaw, A., 2003, "Threedimensional modeling and simulation of weapons effects for obstacle clearance," DoD User Group Conf., Bellevue, Washington, 09-13 June, pp. 9 .

[8] Gooda, Y., 1977, "The analysis on the joint distribution of period and wave height from the records of wave observations (in Japanese)," Technol. Res. Data Estuaries, 272, 1-19.

[9] Liu, Y., Yan, X.-H., Liu, W.T., and Hwang, P.A., 1997, “The probability density function of ocean surface slopes and its effects on radar backscatter," J. Phys. Oceanogr., 27, 782-797.

[10] Cox, C. S., and Munk, W. H., 1954, "Measurement of the roughness of the sea surface from photographs of the sun's glitter," J. Opt. Soc. Amer., 44, 838-850. 
[11] Chu, P.C., and Fan, C.W., 2006. "Prediction of falling cylinder through airwater-sediment columns," AMSE J. Appl. Mech., 73, 300-314.

[12] Chu, P.C., and Fan, C.W., 2007, "Mine impact burial model (IMPACT35) verification and improvement using sediment bearing factor method," IEEE J. Ocean. Eng., 32 (1), pp. 34-48.

[13] Chu, P.C., 2009, "Mine impact burial prediction from one to three dimensions," ASME Appl. Mech. Rev., 62 (1), 010802 (25 pages), DOI: $1115 / 1.3013823$. 